\title{
Dengue fever - an update review and implications for Nigeria, and similar countries
}

\author{
Akaninyene Otu ${ }^{1}$, Bassey Ebenso ${ }^{2}$, Aniekan Etokidem³ $^{3}$, Okechukwu Chukwuekezie ${ }^{4}$
}

1. Department of Internal Medicine, College of Medical Sciences, University of Calabar Nigeria. Tel: +2348105723133. Email: akanotu@yahoo.com

2. Nuffield Centre for International Health and Development, Leeds Institute for Health Sciences, University of Leeds, United Kingdom. Email: B.E.Ebenso@leeds.ac.uk

3. Department of Community Medicine, College of Medical Sciences University of Calabar, Nigeria. Email: etokidem@etokidem.com

4. National Arbovirus and Vectors Research Centre, 33 Park Avenue GRA, Enugu Nigeria. Email: chukwuekezieo@yahoo.com

\begin{abstract}
Background: In the last five decades, dengue has emerged as one of the most important infectious diseases, following a 30-fold increase in global incidence throughout tropical and sub-tropical regions of the world. The actual numbers of dengue cases are under-reported and many cases are misclassified.

Objectives: This article describes the epidemiology, pathophysiology, clinical features and management of dengue. It also explores the implications of infection with this flavivirus for Nigeria, and similar countries.

Methods: The literature search for publications on dengue in West Africa was performed using PubMed, African Journals Online (AJOL), Google Scholar, Web of Science, databases and grey literature to identify all published papers regarding the topic. A snowballing strategy was adopted to identify additional publications.

Results: Recent reports suggest that dengue is a growing public health problem in Nigeria, the magnitude of which needs to be more clearly defined. Nigeria, the most populous country in Africa has an abundance of the Aedes aegypti mosquito which is known to transmit dengue, Zika, as well as chikungunya (CHIKV) and West Nile viruses.

Conclusion: This article provides practical suggestions for strengthening the dengue virus control programme in Nigeria. The Nigerian health system shares similarities with health systems in many other sub-Saharan countries. Therefore, the practical suggestions provided at the end of this review are likely to be applicable to many other African countries.
\end{abstract}

Keywords: Dengue fever update, Nigeria, review.

DOI: https://dx.doi.org/10.4314/ahs.v19i2.23

Cite as: Otu A, Ebenso B, Etokidem A, Chukwnekezie O. Dengue fever - an update and implications for Nigeria. Afri Health Sci.2019;19(2): 2000-2007. bttps://dx.doi.org/10.4314/abs.v19i2.23

\section{Introduction}

Dengue, also known as 'breakbone fever', is the fastest spreading vector-borne viral infection transmitted between humans by the female Aedes aegypti (Ae) mosquito. ${ }^{1-3}$ The infection is caused by one of four dengue virus

$$
\begin{aligned}
& \text { Corresponding author: } \\
& \text { Akaninyene Otu, } \\
& \text { Department of Internal Medicine, } \\
& \text { College of Medical Sciences, } \\
& \text { University of Calabar } \\
& \text { Nigeria. Tel: +2348105723133. } \\
& \text { Email: akanotu@yahoo.com }
\end{aligned}
$$

serotypes (DENV-1, DENV-2, DENV-3 and DENV-4) belonging to the genus flavivirus within the Flaviviridae family. The genus flavivirus also includes pathogens that cause Zika virus disease, yellow fever and West Nile fever. ${ }^{4}$ Dengue virus contains a positive strand RNA with a spherical lipid envelope. The RNA genome codes for envelope, capsid and membrane structural proteins in addition to seven non-structural proteins. Infection with any of the dengue virus sub-types may result either in an asymptomatic infection or a febrile illness of varying severity ranging from mild illness to more severe forms such as dengue hemorrhagic fever (DHF) and dengue shock syndrome (DSS).,6

(C) 2019 Otu et al. Licensee African Health Sciences. This is an Open Access article distributed under the terms of the Creative commons Attribution License (https://creativecommons.org/licenses/BY/4.0), which permits unrestricted use, distribution, and reproduction in any medium, provided the original work is properly cited. 
In the last five decades, dengue has emerged as one of the most important infectious diseases, following a 30fold increase in global incidence throughout tropical and sub-tropical regions of the world. ${ }^{7}$ The actual numbers of dengue cases are under-reported and many cases are misclassified. A recent estimate indicates an annual incidence of 390 million cases of which 96 million $(24.6 \%)$ manifest apparently, with the majority existing as inapparent (asymptomatic) infections. ${ }^{3}$ Dengue poses a risk to 2.53.6 billion people annually in over 125 endemic countries, extending to over 120 million travelers to these countries and is estimated to cause about 10,000 deaths annually. Factors advanced for the extensive global transmission of dengue include rapid population growth, unplanned urbanization, increased international travel, agricultural development and possible global climate changes. ${ }^{8}$ Other contributory factors are ineffective mosquito control measures and limited allocation of resources to public health infrastructure.

This article reviews the literature on dengue with a focus on West Africa. It describes the epidemiology, pathophysiology, clinical features and management of dengue. It also explores the implications of this flavivirus infection for Nigeria, the most populous country in Africa. The dengue virus shares the same vector with other important viruses such as Zika, yellow fever and chikungunya. Improvements in dengue virus control could potentially influence the control of these other arboviral diseases thereby reducing morbidity and mortality attributable to them. The inferences provided at the end of this review are likely to benefit other low-middle income countries that share similar socio-demographic and health system features with Nigeria.

\section{Search strategy and selection criteria}

The literature search for scientific publications on dengue in West Africa was performed using PubMed, African Journals Online (AJOL), Google Scholar, Web of Science, databases and grey literature to identify all relevant published articles on the topic. A snowballing strategy was adopted to identify additional publications. The main search comprised individual searches using detailed medical subject heading (MeSH) terms for dengue, West Africa and Nigeria combined with terms relevant to dengue diagnosis and management. The Boolean operator 'AND' and 'OR' were used to combine and narrow the searches.

\section{Dengue in West Africa and Nigeria}

Among all world regions, West Africa is projected to have the fastest-growing population growth rate over the next half-century. ${ }^{10}$ One recent comparison of dengue risk maps of the African region describes population growth and high rates of poverty in the coastal stretch of West Africa $^{11}$ (between Abidjan and Abuja). This population projection may promote the expansion in dengue incidence in the region if severely weakened health systems ${ }^{12}$ in West African countries fail to prioritize resources for robust surveillance and control of febrile illnesses including dengue. ${ }^{10}$

Clinical reports of dengue in Africa date back to 1926 following an epidemic in Durban, South Africa. ${ }^{13,14}$ However, the dengue virus was only isolated from human sera in West Africa between1960s and 1970s. ${ }^{15,16}$ Specifically, the dengue virus was first isolated in Ibadan, Western Nigeria in $1960 .^{7}$ Although there have been many reports of isolated outbreaks of dengue infection in Nigeria after 1960, it is likely that many outbreaks of dengue may have been neglected, under-recognized or under-reported due to a lack of awareness of health staff and unavailability of diagnostic tools in health institutions. ${ }^{18}$ Recent reports suggest that dengue viruses are a major cause of acute fevers in Nigeria. ${ }^{19,20}$ To highlight the growing importance of dengue in Nigeria, recent seroprevalence surveys conducted in Maiduguri (Northern Nigeria) ${ }^{21}$ and in Ilorin (Western Nigeria) ${ }^{9}$ showed that 10.1\% and $30.8 \%$ of participants respectively, were seropositive for dengue subtype-3 virus (DENV-3). Additionally, dengue IgM sero-prevalence among febrile Nigerian children in South-Western Nigeria has been reported to be $17.2 \%$ and $30.8 \%$ respectively. ${ }^{22,23}$ These high prevalence figures of symptomatic dengue virus infections and of dengue IgM antibodies are indicative of potential endemicity of dengue and of the plausibility of many missed infections by frontline health workers. There is also evidence of high vector density in highly populated Nigerian cities. ${ }^{24}$ These findings together suggest that dengue is a growing public health problem in Nigeria, the magnitude of which needs to be more clearly defined. The diagnosis of dengue in $\mathrm{Ni}$ - 
geria is further complicated by evidence of high co-infection rates of malaria and dengue. A recent report revealed a $10 \%$ active dengue co-infection rate among confirmed malaria cases in Ibadan, South-Western Nigeria. Further assessment of dengue IgG seroprevalence among malaria patients revealed that all the malaria patients in the study were positive for dengue $\operatorname{IgG}$, suggestive of a past dengue infection and consistent with endemicity of dengue virus in this area. ${ }^{25}$ There is a high density of the Aedes aegypti mosquito that transmit dengue, yellow fever (YFV) and chikungunya in Nigeria, and the same applies to the Anopheles mosquito that transmits malaria. ${ }^{26}$

\section{The vectors, transmission, pathophysiology and clinical features}

The vectors of dengue virus are mosquitoes of the genus, Aedes. This genus of mosquito was originally found in tropical and sub-tropical zones, but has now spread to all continents excluding Antarctica. ${ }^{27}$ Aedes aegypti is the primary vector, while Aedes albopictus the Asian tiger mosquito is considered the secondary vector of the disease. Spread of mosquitoes of this genus is largely by manmade activities (international trade in used tyres, lucky bamboo, among others). They breed in transient water collections such as waters in tree holes, or in plant leaf axils, bamboo stumps, rock pools, and artificial containers including tin cans, coconut shells, water storage containers, discarded vehicle tyres, broken earthen and ceramic wares, as well as other water containers around houses. ${ }^{28,29}$ Aedes eggs, which can withstand desiccation, are laid singly on damp substrates just beyond the water line. Hatching may occur within a few minutes to several weeks, depending on a lot of intrinsic and extrinsic factors. Unlike most other mosquitoes, Aedes mosquitoes are active and bite both humans and animals only during the daytime. Early mornings and in the evenings, before dusk, mark their peak biting periods. They may bite several times before completion of a single blood meal. These breeding and biting habits of Aedes aegypti and Aedes albopictus keep them in close proximity to man, facilitating disease transmission. Aedes albopictus is highly adaptive and, thus, can survive in cooler temperate regions. Its spread is facilitated by its tolerance to temperatures below freezing, hibernation, and ability to shelter in microhabitats. Aedes albopictus was originally a South-East Asia mosquito, but has spread to North America, Europe and sub-Saharan Africa. Its presence was established in Nigeria in 1992 by the National Arbovirus and Vectors Research Centre. ${ }^{30}$ Infected humans are the main carriers and multipliers of dengue, and serve as a source of the virus for uninfected mosquitoes. Patients who are already infected with the dengue virus can transmit the infection (for 4-5 days; maximum 12 days) via Aedes mosquitoes after their first symptoms appear. ${ }^{31}$ The female Aedes aegypti mosquito bites multiple people during each feeding period. After an extrinsic incubation period lasting about 4- 10 days, the virus enters the salivary glands of the mosquito, giving the infected mosquito the capacity to transmit the virus for the rest of its life. ${ }^{31}$ Immature dendritic cells in the human skin are the first target with subsequent spread to regional lymph nodes, liver, spleen as well as peripheral blood monocytes. ${ }^{32}$

The syndromes produced by the dengue virus are influenced by age and immunological status. With primary infection, the symptoms are very mild in most children and range from sub-clinical infection or mild undifferentiated febrile syndromes. Secondary infection is associated with a dramatic alteration in the pathophysiology resulting in a greater propensity to develop severe dengue. Clinically, dengue infection may be asymptomatic or manifest with an abrupt onset of fever, headache and malaise 4-5 days after the bite. The patient typically defervesces after 4-5 days of fever with an accompanying generalized maculopapular rash, which signals recovery. Leucopenia may be found at this stage with normal platelet count and transaminase values.

Primary dengue infection induces a lifelong protective immunity to the particular serotype, but cross-reactive immunity to the other serotypes is only partial and temporary. Interestingly, secondary or multiple dengue virus infection has been identified as a major risk factor for severe dengue. ${ }^{33}$ Other risk factors for severe dengue include viral virulence and host genetic background. ${ }^{33}$

For patients who go on to develop DSS, the clinical picture differs after defervescence where they develop tachycardia, bleeding, central cyanosis with cool, clammy extremities. These features have been attributed to a vascular leak which allows plasma extravasation. Activation of the complement and coagulation pathways occur with thrombocytopenia and elevated haematocrit. ${ }^{34}$ Other clinical features include severe and continuous abdominal 
pain, persistent vomiting with haematemesis, hypothermia, restlessness and fainting. Patients may die within the next 24-48 hours if appropriate resuscitative measures are not instituted rapidly. With appropriate resuscitation, other complications such as disseminated intravascular coagulation, multiple organ failure, and respiratory failure can be averted. ${ }^{34}$

\section{Diagnosis}

Early diagnosis of dengue cases is useful in triaging patients. There are four main methods of diagnosing dengue virus infection in exposed persons: i) virus isolation and characterization, ii) detection of genomic sequence by a nucleic acid amplification assay, iii) detection of dengue virus-specific antibodies and iv) identification of dengue virus antigens (glycoproteins). Virus isolation is achieved by cell culture which gives the most specific test result. ${ }^{1}$ Sera for virus isolation is usually collected in the first 3-5 days of fever. Isolation of the virus depends largely on viral load thus limiting the period within which the dengue virus can be successfully isolated in patient serum. ${ }^{16,35}$ Viral cell culture has low sensitivity, it is a laborious and time consuming procedure. Viral identification can be done using dengue-specific monoclonal antibodies in immunofluorescence and reverse transcription-polymerase chain reaction (RT-PCR).

Serologic assays are relatively inexpensive and easy to perform. These characteristics make them the most commonly used tests for dengue infection. IgM levels begin to rise by the third day of a primary infection and peak at 2 weeks after the onset of fever. IgG is detectable at the end of the first week of illness and can persist for life. ELISA tests can assay for IgM and IgG levels and the Ig$\mathrm{M}: \operatorname{IgG}$ ratio is useful in distinguishing primary from secondary dengue virus infections. IgM:IgG ratios of greater than 1.2 or 1.4 are indicative of primary dengue infection while IgM:IgG ratios of less than 1.2 or 1.4 are indicative of secondary dengue infection. ${ }^{36}$ The potential cross reactivity of dengue virus with other flaviviridae when tested with serologic assays remains a significant limitation to its use. Prior yellow fever vaccination can also give rise to a false positive serologic test for dengue virus. The extended sero-conversion period also gives rise to false negative tests. $^{37}$

The dengue virus antigens in tissues such as the liver, spleen and lymph nodes can be identified using an enzyme and a colorimetric substrate with antibodies. The flaviviruses all produce a glycoprotein called NS1. Tests such as antigen-capture ELISA and lateral flow antigen detection can be utilized to identify the presence of these glycoproteins in the blood stream. ${ }^{1}$ The NS1 antigen is detectable from days 0-9 after the onset of symptoms though detection appears to be higher in samples collected up to three days after onset of symptoms. The rapid dipstick test, the Dengue NS1 Ag Strip (Bio-Rad Laboratories, Marnes-la-Coquette, France), is now available and this provides results within 15 minutes. The NS1 dipstick test has been shown to have a significantly higher sensitivity for primary infections $(94.7 \%)$ than for secondary infections $(67.1 \% ; \mathrm{P}<0.001){ }^{38,39}$ The NS1 protein tests now appear to be a potential alternative to culture, PCR and serology. ${ }^{40}$

\section{Treatment, prevention and control}

There is no specific treatment for dengue fever other than supportive measures and judicious fluid therapy. ${ }^{8}$ In severe dengue, maintenance of the patient's body fluid volume is critical. ${ }^{31}$ If the patient is appropriately managed during the critical period which is between 48-72 hours, the likelihood of death is minimal. ${ }^{41}$ Early notification of cases of dengue is vital in establishing a robust response to disease outbreaks.

The global strategy for dengue prevention and control has five elements: i) vector control, ii) active disease surveillance based on a comprehensive health information system, iii) emergency preparedness, iv) capacity building and v) vector control research. ${ }^{2}$ In December 2015, the vaccine CYD-TDV (marketed as Dengvaxia ${ }^{\circledR}$ ), developed by the French pharmaceutical company Sanofi, was approved for use in Brazil, Mexico and the Philippines. ${ }^{42}$ Dengvaxia is a live attenuated tetravalent vaccine ${ }^{43}$ developed from the yellow fever virus ${ }^{44}$ to stimulate the body's immune system into making antibodies against all four subtypes of dengue. The Yellow fever virus and dengue virus have the same genus. For Dengvaxia however, the virus is genetically engineered to include genes encoding for dengue proteins. However, a warning was released by Sanofi on Nov 29, 2017, that Dengvaxia could increase the risk of severe dengue in particular circumstances, following a review of the data from the clinical trials. ${ }^{45}$ 
This led to a suspension of the dengue vaccination programme in the Philippines. Another vaccine candidate the Takeda's Tetravalent Dengue Vaccine (TDV) is currently undergoing phase 2 trials. ${ }^{45}$ A recent article that reviewed optimal control strategies for dengue during the outbreak in Pakistan described a strong reciprocal relationship between the use of insecticide and vaccination; as the cost of insecticide increased the use of vaccination appeared to increase also. ${ }^{46}$

\section{Implications for Nigeria, and similar countries}

1. To complement sporadic reports of dengue prevalence by individual scholars in the country, the Federal Ministry of Health in Nigeria should, as a matter of urgency, invest in nationwide dengue surveys to determine the magnitude of different dengue serotypes circulating in Nigeria, following which dengue should be made a reportable disease in the country. The nationwide dengue surveys should run simultaneously with vector surveillance and the concomitant virus identification assays, to explicitly define the dengue serotypes and their vectors in the country. A robust and integrated dengue disease surveillance system needs to be established and linked to the national health information system, with a set of core indicators which should be monitored. Outbreak preparedness involving the relevant agencies should be developed as a matter of priority.

2. There is a need for national authorities to strengthen dengue fever control strategies as dengue is one of many diseases that is routinely misdiagnosed as malaria and thus remains a neglected tropical disease in the country. Vector control and education about basic ways of limiting bites from the vectors of dengue by use of protective clothing needs to be prioritized. Source reduction is crucial and should include environmental modification strategies such as fitting lids or covers on water containers and disposing of such containers where possible. The use of insecticides such as organophosphates (temephos) and pyrethroids has been shown to be beneficial though resistance of both Aedes aegypti and Aedes albopictus to these has been described. The use of genetically modified (GM) mosquitoes has been shown to reduce the population of disease carrying vectors by $95 \% .{ }^{47}$ The GM mosquitoes work by two methods namely, "population replacement" in which a mosquito population is substituted by one that is unable to transmit pathogens. A bacterial symbiont known as Wolbachia has the capacity to infect the mosquito and limit its ability to spread other pathogens. The second approach is called "population suppression" and this can be achieved by the use of the Sterile Insect Technique (SIT) where sterile male mosquitoes are produced to mate with wild female ultimately leading to a reduction in the mosquito population. ${ }^{48}$ Such an effective strategy should be exploited. Given the rapid unplanned nature of urban development and the poor water supply and sewage systems in Nigeria ${ }^{44}$, greater efforts at vector control are likely to yield a great impact. The overall benefits of vector control are likely to influence the transmission of other diseases such as malaria, yellow fever, Zika and chikungunya.

3. We recommend a more comprehensive focus on, and commitment to, improved diagnosis of febrile illnesses ${ }^{49}$ in Nigeria. Laboratory surveillance of dengue is vital as this will enhance the identification and confirmation of dengue given that malaria, yellow fever, chikungunya and dengue may be clinically indistinguishable causes of fever. ${ }^{50}$ The provision of NS1 dipstick testing for dengue at the primary health care level might be a bold step in the right direction. The NS1 dipstick test is relatively easy to use, it has an acceptable sensitivity and specificity profile and provides rapid results.

4. Building the capacity of frontline health workers and prioritizing dengue as a cause of fever in our setting is critical for success. An intensive push for health worker training and utilization of clinical algorithms for managing dengue is likely to yield dividends. ${ }^{51}$ This capacity building should be extended to laboratory scientists and entomologists to promote rapid and accurate diagnosis of dengue infections across Nigeria while ramping up vector identification and control.

5. In the context of ongoing concerns over the spread of Zika from the Americas to other regions of the world, Nigeria needs to be on the alert for cases of Zika virus disease ${ }^{4}$, especially as the country is within the dengue belt where both the Aedes aegypti (i.e. the vector for both dengue and the Zika) and Aedes albopictus mosquitoes abound. Surveillance and monitoring for Zika cases is a necessity in addition to appropriate mosquito population 
control measures. As intrauterine, sexual intercourse, and blood-borne spread of Zika are now proven means of transmission, appropriate precautions should be widely promoted in our communities.

\section{Conflict of interest}

All authors declare they have no conflict of interest.

\section{References}

1. Rodríguez-Barraquer I, Solomon SS, Kuganantham P, Srikrishnan AK, Vasudevan CK, Iqbal SH, et al. The Hidden Burden of Dengue and Chikungunya in Chennai, India. PLoS Negl Trop Dis. 2015; 9:e0003906.

2. Guzman MG, Halstead SB, Artsob H, Buchy P, Farrar J, Gubler DJ, et al. Dengue: a continuing global threat. Nature Reviews Microbiology [serial on the Internet]. 2010; S7-S16: Available from: http://www.nature.com/nrmicro/journal/v8/n12_supp/full/nrmicro2460.html.

3. Bhatt S, Gething PW, Brady OJ, Messina JP, Farlow AW, Moyes CL, et al. The global distribution and burden of dengue. Nature. 2013; 496:504-7.

4. Gulland A. WHO urges countries in dengue belt to look out for Zika. BMJ. 2016; 352.

5. Gubler DJ. Epidemic dengue/dengue hemorrhagic fever as a public health, social and economic problem in the $21^{\text {st }}$ century. Trends in Microbiology. 2002; 10:100-3.

6. Kosasih H, Alisjahbana B, Nurhayati, de Mast Q, Rudiman IF, Widjaja S, et al. The Epidemiology, Virology and Clinical Findings of Dengue Virus Infections in a Cohort of Indonesian Adults in Western Java. PLoS Negl Trop Dis. 2016; 10:e004390.

7. WHO. global strategy for dengue prevention and control 2012-2020. Geneva: WHO2012.

8. Gubler DJ. Dengue, Urbanization and Globalization: The Unholy Trinity of the 21(st) Century. Tropical Medicine and Health. 2011; 39:3-11.

9. Guzman MG, Kouri G. Dengue: an update. The Lancet Infectious diseases. 2002; 2:33-42.

10. United-Nations. World Urbanization Prospects: The 2010 Revision. New York: United Nations Population Division, Department of Economic and Social Affairs; 2011 [cited 201621 February]; Available from: http:// esa.un.org/unpd/wup/.

11. Rogers DJ, Suk JE, Semenza JC. Using global maps to predict the risk of dengue in Europe. Acta Trop. 2014; 129:1-14.

12. Otu A, Ebenso B, Okuzu O, Osifo-Dawodu E. Using a mHealth tutorial application to change knowledge and attitude of frontline health workers to Ebola virus disease in Nigeria: a before-and-after study. Human Resources for Health. 2016; 14:1-9.

13. Gubler DJ. Dengue. In: Monath TPM, Raton B, editors. Epidemiology of Arthropod Borne Viral Disease. FL: CRC Press; 1988. p. 223-60.

14. Johnson BK, Musoke S, Ocheng D, Gichogo A, Rees PH. Dengue-2 Virus in Kenya. The Lancet. 320:208-9.

15. Carey DE, Causey OR, Reddy S, Cooke AR. Dengue Viruses from febrile patients in Nigeria, 1964-68. The Lancet. 297:105-6.

16. Fagbami AH, Monath TP, Fabiyi A. Dengue virus infections in Nigeria: a survey for antibodies in monkeys and humans. Trans R Soc Trop Med Hyg. 1977; 71:60-5.

17. Amarasinghe A, Kuritsky JN, Letson GW, Margolis HS. Dengue Virus Infection in Africa. Emerging Infectious Diseases. 2011; 17:1349-54.

18. Baba MM, Talle M. The Effect of Climate on Dengue Virus Infections in Nigeria. New York Science Journal. 2011; 4:28-33

19. Ayukekbong JA. Dengue Virus in Nigeria: Current Status and Future Perspective. British Journal of Virology. 2014; 1:106-11.

20. Okogun GR, Nwoke B, Okere A, Anosike J, Esekhegbe A. Epidemiological implications of preferences of breeding sites of mosquito species in Mid-Western Nigeria. Annals of Agricultural and Environmental Medicine: AAEM. 2003; 10:217-22.

21. Idris AN, Baba MM, Thairu Y, Bamidele O. Sero-prevalence of dengue type-3 Virus among patients with febrile illnesses attending a tertiary hospital in Maiduguri, Nigeria. Int J Med Med Sci. 2013; 5:560-3.

22. Adedayo F, I. Nioma, M.B. Olanrewaju, A. Adeyinka and A. Ebele,. Serological evidence of recent dengue virus infection among febrile children in a semi arid zone. Am J Infect Dis. 2013; 9:7-10.

23. Oladipo EK, Amanetu C, Gbadero TA, Oloke JK. Detectable anti-dengue virus $\operatorname{IgM}$ antibodies among healthy individuals in Ogbomoso, Oyo state, Nigeria. Am J Infectious Dis. 2014; 10:64-7.

24. Adeleke MA, Mafiana CF, Idowu AB, Sam-Wobo SO, Idowu OA. Population dynamics of indoor sampled mosquitoes and their implication in disease transmission in Abeokuta, South-Western Nigeria. Journal of Vector Borne Diseases. 2010; 47:33-8.

25. Oyero OG, Ayukekbong JA. High dengue NS1 anti- 
genemia in febrile patients in Ibadan, Nigeria. Virus Research. 2014; 191:59-61.

26. Baba M, Logue $\mathrm{CH}$, Oderinde B, Abdulmaleek $\mathrm{H}$, Williams J, Lewis J, et al. Evidence of arbovirus co-infection in suspected febrile malaria and typhoid patients in Nigeria. Journal of Infection in Developing Countries. 2013; 7:51-9.

27. Benedict MQ, Levine RS, Hawley WA,1 Lounibos LP. Spread of the tiger: global risk of invasion by the mosquito Aedes albopictus. Vector Borne Zoonotic Dis. 2007;7:76-85.

28. Onyido AE, Ezike VI, Ozumba NA, Nwosu EO, Ikpeze OO, Obiukwu MO, et al. Crepuscular Man-Biting Mosquitoes Of A Tropical Zoological Garden In Enugu, South-Eastern Nigeria. Internet Journal of Parasitic Diseases. 2008; 4:3-.

29. Irikannu KC, Chukwuekezie OC. Malaria and Man-biting Mosquitoes in Tropical Africa: Omniscriptum Gmbh \& Company Kg.; 2015.

30. Savage HM, Ezike VI, Nwankwo AC, Spiegel R, Miller BR. First record of breeding populations of Aedes albopictus in continental Africa: implications for arboviral transmission. Journal of the American Mosquito Control Association. 1992; 8:101-3.

31. WHO. Dengue and severe dengue: Fact sheet $\mathrm{N}^{\circ} 117$. Gevena: WHO; 2015 [cited 201620 February]; Available from: http://www.who.int/mediacentre/factsheets / fs117/en/.

32. Jessie K, Fong MY, Devi S, Lam SK, Wong KT. Localization of Dengue Virus in Naturally Infected Human Tissues, by Immunohistochemistry and In Situ Hybridization. Journal of Infectious Diseases. 2004; 189:1411-8.

33. Bravo JR, Guzman MG, Kouri GP. Why dengue haemorrhagic fever in Cuba? 1. Individual risk factors for dengue haemorrhagic fever/dengue shock syndrome (DHF/DSS). Trans R Soc Trop Med Hyg. 1987; 81:816-20.

34. WHO. Dengue: Guidelines for diagnosis, treatment, prevention and control. New edition. Geneva: WHO and and the Special Programme for Research and Training in Tropical Diseases. (TDR); 2009.

35. Back, AT, Lundkvist a. 2013. Dengue viruses - an overview. Infection Ecology \& Epidemiology. 2013; 3

36. Shu P-Y, Chen L-K, Chang S-F, Yueh Y-Y, Chow L, Chien L-J, et al. Comparison of Capture Immunoglobulin M (IgM) and IgG Enzyme-Linked Immunosorbent Assay (ELISA) and Nonstructural Protein NS1 Sero-
type-Specific IgG ELISA for Differentiation of Primary and Secondary Dengue Virus Infections. Clinical and Diagnostic Laboratory Immunology. 2003; 10:622-30.

37. Schwartz E, Mileguir F, Grossman Z, Mendelson E. 2000. Evaluation of ELISA-based sero-diagnosis of dengue fever in travelers. Journal of clinical virology : the official publication of the Pan American Society for Clinical Virology. 2000; 19: 169-173.

38. Tricou V, Vu HT, Quynh NV, Nguyen CV, Tran HT, Farrar J, Wills B, Simmons CP. Comparison of two dengue NS1 rapid tests for sensitivity, specificity and relationship to viraemia and antibody responses. BMC Infect Dis. 2010;10:142.

39. Hang VT, Nguyet NM, Trung DT, Tricou V, Yoksan S, Dung NM, Van Ngoc T, Hien TT, Farrar J, Wills B, Simmons CP. Diagnostic accuracy of NS1 ELISA and lateral flow rapid tests for dengue sensitivity, specificity and relationship to viraemia and antibody responses. PLoS Negl Trop Dis. 2009;3:e360.

40. Wang SM, Sekaran SD. 2010. Evaluation of a commercial SD dengue virus NS1 antigen capture enzyme-linked immunosorbent assay kit for early diagnosis of dengue virus infection. Journal of Clinical Microbiology. 2010; 48: 2793-2797

41. Rajapakse S, Rodrigo C, Rajapakse A. Treatment of dengue fever. Infection and Drug Resistance. 2012; 5:103-12. 42. Maron DF. First Dengue Fever Vaccine Gets Green Light in 3 Countries. New York: Scientific American 2015 [cited 201620 February]; Available from: http:// www.scientificamerican.com/article/first-dengue-fevervaccine-gets-green-light-in-3-countries/.

43. WHO. Vaccine Satety Basics e-learning course: Live Attenuated Vaccines (LAV). Geneva: WHO; 2016 [cited 201620 February]; Available from: http://vaccine-safety-training.org/live-attenuated-vaccines.html.

44. McCall PJ, Lenhart A. Dengue control. The Lancet infectious diseases. 2008; 8: 7-9.

45. Editorial. The dengue vaccine dilemma. The Lancet Infectious Diseases. 2018; 18(2): 123.

46. FB Agusto, MA Khan. Optimal control strategies for dengue transmission in Pakistan, Math biosci. 2018; 305: 102-121

47. Carvalho DO, McKemey AR, Garziera L, Lacroix R, Donnelly C, Alphey L et al. Suppression of a field population of Aedes aegypti in Brazil by sustained release of transgenic male mosquitoes. PLoS Negl Trop Dis. 9(7): e0003864. 
48. Qsim M, Ashfaq UA, Yousaf MZ, Masoud MS, Rasul I, Noor N, Hussain A. Genetically Modified Aedes aegypti to Control Dengue: A Review. Crit Rev Eukaryot Gene Expr. 2017;27(4):331-340. doi: 10.1615/Crit Rev Eukaryot Gene Expr. 2017019937.

49. Stoler J, al Dashti R, Anto F, Fobil JN, Awandare GA. Deconstructing "malaria": West Africa as the next front for dengue fever surveillance and control. Acta Tropica. 2014; 134:58-65.
50. Nkoghe D, Kassa RFK, Bisvigou U, Caron M, Grard G, Leroy EM. No clinical or biological difference between Chikungunya and Dengue Fever during the 2010 Gabonese outbreak. Infectious Disease Reports. 2012; 4:e5. 51. Eisenhut M. Diagnosis of Viral Hemorrhagic Fevers in Travelers Returning From West Africa. Journal of Travel Medicine. 2013; 20:63-. 